\title{
Ex vivo differential phase contrast and magnetic resonance imaging for characterization of human carotid atherosclerotic plaques
}

\author{
Romana Meletta $^{1} \cdot$ Nicole Borel $^{2} \cdot$ Paul Stolzmann $^{3} \cdot$ Alberto Astolfo $^{4}$. \\ Jan Klohs ${ }^{5} \cdot$ Marco Stampanoni $^{4} \cdot$ Markus Rudin $^{5} \cdot$ Roger Schibli $^{1}$. \\ Stefanie D. Krämer ${ }^{1} \cdot$ Adrienne Müller Herde $^{1}$
}

Received: 10 April 2015/Accepted: 6 July 2015/Published online: 16 July 2015

(C) Springer Science+Business Media Dordrecht 2015

\begin{abstract}
Non-invasive detection of specific atherosclerotic plaque components related to vulnerability is of high clinical relevance to prevent cerebrovascular events. The feasibility of magnetic resonance imaging (MRI) for characterization of plaque components was already demonstrated. We aimed to evaluate the potential of ex vivo differential phase contrast $\mathrm{X}$-ray tomography (DPC) to accurately characterize human carotid plaque components in comparison to high field multicontrast MRI and histopathology. Two human plaque segments, obtained from carotid endarterectomy, classified according to criteria of the American Heart Association as stable and unstable plaque, were examined by ex vivo DPC tomography and multicontrast MRI (T1-, T2-, and proton density-weighted imaging, magnetization transfer contrast, diffusion-weighted imaging). To identify specific plaque
\end{abstract}

Electronic supplementary material The online version of this article (doi:10.1007/s10554-015-0706-y) contains supplementary material, which is available to authorized users.

Adrienne Müller Herde

adrienne.herde@pharma.ethz.ch

1 Department of Chemistry and Applied Biosciences of ETH Zurich, Center for Radiopharmaceutical Sciences ETH-PSIUSZ, Vladimir-Prelog-Weg 1-5/10, 8093 Zurich, Switzerland

2 Institute of Veterinary Pathology, University of Zurich, Winterthurerstrasse 268, 8057 Zurich, Switzerland

3 Department of Medical Imaging, University Hospital Zurich, Raemistrasse 100, 8006 Zurich, Switzerland

4 Swiss Light Source, Paul Scherrer Institute, 5232 Villigen-PSI, Switzerland

5 Institute for Biomedical Engineering, and Center for Neuroscience Research, University and ETH Zurich ETH Zurich, Wolfgang-Pauli-Str. 27, 8093 Zurich, Switzerland components, the plaques were subsequently sectioned and stained for fibrous and cellular components, smooth muscle cells, hemosiderin, and fibrin. Histological data were then matched with DPC and MR images to define signal criteria for atherosclerotic plaque components. Characteristic structures, such as the lipid and necrotic core covered by a fibrous cap, calcification and hemosiderin deposits were delineated by histology and found with excellent sensitivity, resolution and accuracy in both imaging modalities. DPC tomography was superior to MRI regarding resolution and soft tissue contrast. Ex vivo DPC tomography allowed accurate identification of structures and components of atherosclerotic plaques at different lesion stages, in good correlation with histopathological findings.

Keywords X-rays - Synchrotron - Differential phase contrast - Magnetic resonance imaging - Carotid plaque . Atherosclerosis

$\begin{array}{ll}\text { Abbreviations } \\ \text { DPC } & \text { Differential phase contrast } \\ \text { MRI } & \text { Magnetic resonance imaging } \\ \text { PD } & \text { Proton density } \\ \text { MTC } & \text { Magnetization transfer contrast } \\ \text { DW } & \text { Diffusion-weighted } \\ \text { L } & \text { Lumen of the blood vessel } \\ \text { M } & \text { Media } \\ \text { Int } & \text { Intima } \\ \text { IT } & \text { Intimal thickening } \\ \text { FC } & \text { Fibrous cap } \\ \text { LC } & \text { Lipid core } \\ \text { NC } & \text { Necrotic core } \\ \text { U } & \text { Ulceration site }\end{array}$




\section{Introduction}

The rupture of atherosclerotic plaques in carotid arteries is the main cause for stroke. Every year, 15 million people suffer a stroke worldwide. One-third of the affected die, one-third are left permanently disabled and one-third will recover completely from their attack [1]. Projections show that by the year 2030, an additional 3.4 million people aged $\geq 18$ years will have had a stroke corresponding to $20.5 \%$ increase in prevalence from 2012 [2]. To prevent stroke, the most frequently performed surgical intervention is carotid endarterectomy.

Most ruptures occur in so called unstable plaques containing a highly inflamed lipid-rich and/or necrotic core covered by a thin fibrous cap [3]. Early identification and characterization of plaques are crucial for risk prediction and prevention of adverse events. Invasive techniques, such as intravascular ultrasound, optical coherence tomography, and carotid angiography can reveal luminal stenosis, wall thickness and plaque volume. However, plaque rupture and stroke are not dependent on the extent of stenosis. Characterizing plaque morphology and, in particular, plaque stabilizing and destabilizing components may provide more information on plaque instability and consequently on the risk of rupture. Besides molecular imaging such as positron emission tomography (PET) and single photon emission computed tomography (SPECT), the two imaging techniques computed tomography (CT) and magnetic resonance imaging (MRI) that are widely accepted by the medical community, have the potential to assess plaque vulnerability non-invasively. However, to detect density differentials within soft tissues, such as in atherosclerotic plaques, a good sensitivity coupled with high-resolution are required, which does not apply to conventional $\mathrm{CT}$ and MRI. A recently introduced technique, synchrotron-based differential phase contrast X-ray (DPC) tomography [4-6], does not only measure the attenuation of X-rays passing through tissue, as in standard X-ray tomography, but in addition it measures the phase shift caused by refraction, which the coherent X-ray beam undergoes when passing through tissue with different refractive indices. This results in a high sensitivity to electron density differences and thereby improving contrast for soft tissue. The objective of our study was to evaluate the feasibility of ex vivo DPC tomography to morphologically characterize two human carotid atherosclerotic plaque specimens. Multicontrastweighted MRI is capable of imaging vessel wall structures and carotid plaque compositions in vivo [7] and ex vivo [8]. MRI differentiates plaque components on the basis of water content, physical state, and molecular motion or diffusion while providing information-rich images at high spatial resolution. The most common strategy is to analyze multicontrast images (T1-, T2-, proton density (PD)weighted imaging) that reveal components with different contrast [9]. The content and distribution of lipids is an important aspect of atherosclerotic plaques. To improve the contrast between lipids and fibrous tissue, the low mobility of protons bound to lipids has been exploited by using diffusion-weighted (DW) imaging [10]. For better identification of protein-rich regions magnetization transfer contrast (MTC) was recommended by Qiao et al. [11].

To our knowledge no direct comparison of DPC images, multicontrast MR images and histopathology of human endarterectomized carotid plaques has been carried out. The purpose of this ex vivo study was (1) to explore the potential of a grating interferometer at a synchrotron X-ray source to measure the DPC between different components in human stable and unstable carotid plaques, (2) to compare the images with multicontrast high-resolution MRI and (3) to correlate the generated images from both modalities with histopathology.

\section{Methods}

\section{Study design and experimental overview}

The study was carried out in concordance with the Helsinki declaration. Written informed consent was obtained from a 75-year-old male patient scheduled for carotid endarterectomy. Two carotid specimens, one originating from the Arteria carotis externa and the other from the Arteria carotis communis/interna, were immediately transferred into RNAlater ${ }^{\circledR}$ (Sigma, St. Louis, USA) solution and stored at $4{ }^{\circ} \mathrm{C}$ overnight according to our standardized procedure for plaque harvesting and bio-banking [12]. The next day, tissues were fixed in $4 \%$ formalin for $24 \mathrm{~h}$ at $4{ }^{\circ} \mathrm{C}$ and finally stored in PBS at $4{ }^{\circ} \mathrm{C}$.

First, the two specimens were scanned by DPC tomography at the TOMCAT (Tomographic Microscopy and Coherent Radiology Experiments) beamline of the Swiss Light Source, followed by imaging on a small animal MR system using different MR contrasts. In a second step, both plaques were characterized by histological workup and, finally, the DPC and MR images were matched with the corresponding histological sections.

\section{Differential phase contrast imaging and three- dimensional rendering}

The presented data sets were obtained by synchrotronbased X-ray tomography using the differential phase contrast technique $[4,6]$ at a photon energy of $25 \mathrm{keV}$. Carotid artery segments were placed in a falcon tube filled with 
PBS. To measure the phase shift, a grating interferometer was used and series of projection images were taken while the carotid plaque specimens rotated. A $300 \mu \mathrm{m}$ thick LAG:Ce scintillator converted the X-rays to visible light, which were captured by a CMOS camera (PCO.Edge, PCO AG, Kelheim, Germany). The total scan time for a segment with a field of view (FOV) of $12 \mathrm{~mm} \times 3.5 \mathrm{~mm}$ was about $90 \mathrm{~min}$. More details about components and parameters are provided in Table S1.

To obtain 3-dimensional images of the DPC data sets, individual Tiff files were converted to a multilayer Tiff file and processed by Imaris software (Bitplane AG, Zurich, Switzerland).

\section{Magnetic resonance imaging}

MRI was performed with a Bruker Pharmascan 7/16 small animal MR system equipped with a gradient system capable of a maximum gradient strength of $760 \mathrm{mT} / \mathrm{m}$, with a $80 \mu$ s rise time and a quadrature birdcage resonator. The specimens were placed in a Falcon tube, filled with PBS, inside the volume resonator and kept at $24{ }^{\circ} \mathrm{C}$. Reference data was acquired in coronal and sagittal orientations for accurate positioning of the plaque specimen. Before imaging a fieldmap-based local shimming was performed on the specimen using the automated MAPshim routine to reduce field inhomogeneities. The imaging protocol consisted of different spin echo (SE) sequences using different dimensions of the FOVs, matrix sizes, echo times (TE) and repetition times (TR) as shown in Table S2. For all sequences 30 averages were performed.

To calculate the MTC, images with ( $\left.\mathrm{MT}_{\text {on }}\right)$ and without $\left(\mathrm{MT}_{\text {off }}\right)$ the application of a saturation pulse were acquired [11]. For MT offset the frequency and amplitude were optimized and a Gaussian saturation pulse with an offset frequency of $3.5 \mathrm{kHz}$ and amplitude of $30 \mu \mathrm{T}$ was found optimal. MT subtraction maps were calculated using the equation:

$\mathrm{MTC}=\mathrm{MT}_{\text {on }}-\mathrm{MT}_{\text {off }}$.

For DW imaging, diffusion-encoding was applied (gradient pulse duration $=2.5 \mathrm{~ms}$, gradient pulse separation $=8.1 \mathrm{~ms})$ with a b-value of $650 \mathrm{~s} / \mathrm{mm} \mathrm{[2]}$.

\section{Histological processing and histopathology}

After DPC and MRI examinations, the two carotid plaques were paraffin-embedded and serial sections of $2.5 \mu \mathrm{m}$ were prepared for further histological and immunohistochemical investigations. Sections were routinely stained with hematoxylin and eosin (HE), Masson's trichrome, Elastica van Gieson (VG-Elastica), phosphotungstic acid hematoxylin (PTAH) showing fibrin deposits in lesions, and
Prussian blue staining to identify iron-containing hemosiderin from previous hemorrhage. For immunohistochemistry, the monoclonal antibody anti-human alpha smooth muscle cell actin (anti-SMA, 1:400, mouse, M0851, Dako, Baar, Switzerland) was used. The detection system included the Dako RealKit (Dako) on the immunostainer (Dako). All sections were digitized by a slide scanner with a pixel size of $0.221 \mu \mathrm{m} \times 0.221 \mu \mathrm{m}$ (Pannoramic 250, 3D Histech, Sysmex, Horgen, Switzerland). Pathological classification of the two plaque types was done in a first instance macroscopically by the surgeon (Z.R.) according to their surface morphology and further investigated on the basis of the modified American Heart Association (AHA)-classifications [13] by a board-certified pathologist (N.B.) using histology and specific staining methods. The experienced pathologist (N.B.) identified vessel layers and plaque components, such as media (M), intima (Int), intimal thickening (IT), fibrous cap (FC), lipid core (LC), necrotic core (NC), inflammatory cell infiltration, hemosiderin deposits, neovascularization, and calcifications.

\section{Interpretation of DPC and MRI data}

The DPC (stable or unstable plaque: 580 slices), MR (stable plaque 25 slices; unstable plaque 40 slices) and histological images (stable plaque 36 slices; unstable plaque 180 slices) were manually matched (Tiff files, Adobe Photoshop CS6 extended, Version 13.0.1) using the known location and distance between DPC images, MR images and histological cross sections. Furthermore, gross morphological features, such as plaque shape, vessel wall thickness and shape as well as calcium deposits were used to optimize the matches. We did not account for shrinkage of the specimen caused by histological processing, as it can vary across the specimen and would require multiple landmarks for accurate matching [14]. The above mentioned plaque components were identified in both imaging modalities using the histological sections as reference. Three independent investigators (R.M., P.S., A.M.H.) analyzed DPC and MR images for plaque signal characteristics in comparison with surrounding structures.

\section{Results}

\section{Morphological characterization of a stable and unstable plaque by histology}

According to histopathology, the specimen originating from the Arteria carotis externa was classified as a stable plaque (Fig. 1a) and the specimen obtained from Arteria carotis communis/interna as an unstable plaque (Fig. 1b). 


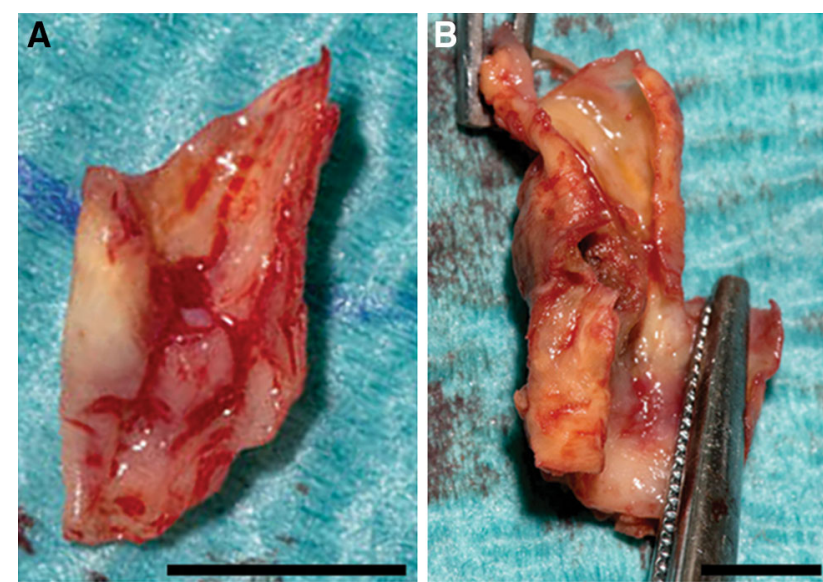

Fig. 1 Whole mount human endarterectomized carotid plaques. a Stable plaque excised from Arteria carotis externa. b Unstable plaque excised from Arteria carotis communis/interna. Scale bars $0.5 \mathrm{~cm}$

Histological workup was performed at the end of the ex vivo imaging procedures. Figure 2 shows the histological identification of the stable and unstable lesions. With $\mathrm{HE}$, the investigated stable plaque presented a thick fibrous cap with fibrillar eosinophilic material. A high collagen content (blue) and local accumulation of erythrocytes (red) between the fibrous cap and the intima was proven by Masson's trichrome staining. A staining for iron-containing hemosiderin (Prussian blue) was negative. Elastic fibers, stained black with VG-Elastica, were present in the lamina elastica interna and media. Immunohistochemical analyses using an anti-SMA antibody demonstrated the presence of smooth muscle cells (SMC) in the lamina elastica interna and media.

The unstable plaque presented towards one plaque shoulder a large necrotic core overlaid by a thicker fibrous cap and towards the other plaque shoulder a smaller lipid core overlaid by a thinner fibrous cap. Invasion of many macrophages and immune cells into the lipid core and partially necrotic core was strongly indicated by HE. The necrotic core contained cell debris, necrotic material and cholesterol crystals. A locally extensive calcification (arrow) was clearly detected with HE. Erythrocytes in the lipid core were of intensive red and SMCs in the media were stained light red by Masson's trichrome. Intracellular hemosiderin pigments, a sign of erythrophagocytosis indicating preceding plaque hemorrhage, were found in macrophages in the lipid core (Fig. 2a high-power magnification, Prussian blue). Positive Prussian blue staining in the media (Fig. 2b, high-power magnification) is linked to the ability of SMCs to phagocytose hemederived iron $[15,16]$. The staining of SMCs correlated with the presence of elastic fibers in the media and lamina elastica interna. Few SMCs were also present in regions of the fibrous cap. PTAH staining identified intraplaque fibrin in the lipid core and necrotic core of the unstable plaque. Fibrin deposition in lipid core/necrotic core regions are known to delineate late stage plaques [17]. Furthermore PTAH staining revealed a ruptured fibrous cap as visualized by a fibrin-positive ulceration site $(\mathrm{U})$ towards the lumen of the blood vessel.

\section{Appearances of atherosclerotic plaque components in DPC tomography and multicontrast MRI}

In the following section we demonstrate the similarities and differences in appearance of plaque components among the different imaging modalities and in correlation with histopathology. Figure 3a and Figures S1 and S2 show the matched DPC images, MR images and histopathology sections of the stable and unstable carotid plaque. Visual appearances of each plaque component in DPC and MR tomography are summarized in Table 1 . The high-resolution and sensitivity of DPC tomography enabled highpower magnification images which are shown with the matching HE stained sections in Fig. 3b-k. Animated Giffiles of the DPC scans are provided in Video S1 and S2.

\section{Tunica media}

Histopathology revealed SMCs and elastic fibers in the media of the stable and unstable plaque. The stable plaque displayed a media of homogeneous texture in DPC and MR images (Fig. 3a, c). Hyperintense (bright signal) T1weighted and DW images distinguished the media from the intima. In contrast, media of the unstable plaque displayed hypointense (dark signal) T1-, T2-, DW-, and PD-weighted images, hyperintense MTC images, and an isodense signal in DPC images. Staining for hemosiderin disclosed blue precipitates as iron-containing particles engulfed by SMCs in the media of the unstable plaque which account for prominent signal intensities.

\section{Tunica intima}

The stable plaque consisted of an intact intima, with a homogeneous texture and intermediate intensity in most image contrasts, except for MTC and DW images where they appeared hypointense and hyperintense, respectively (Fig. 3a, c).

\section{Intimal thickening}

The intimal thickening in the stable plaque appeared homogenous and hyperdense in DPC delimiting it from the intima. MTC images revealed a hypointense intimal thickening (Fig. 3a, d). 


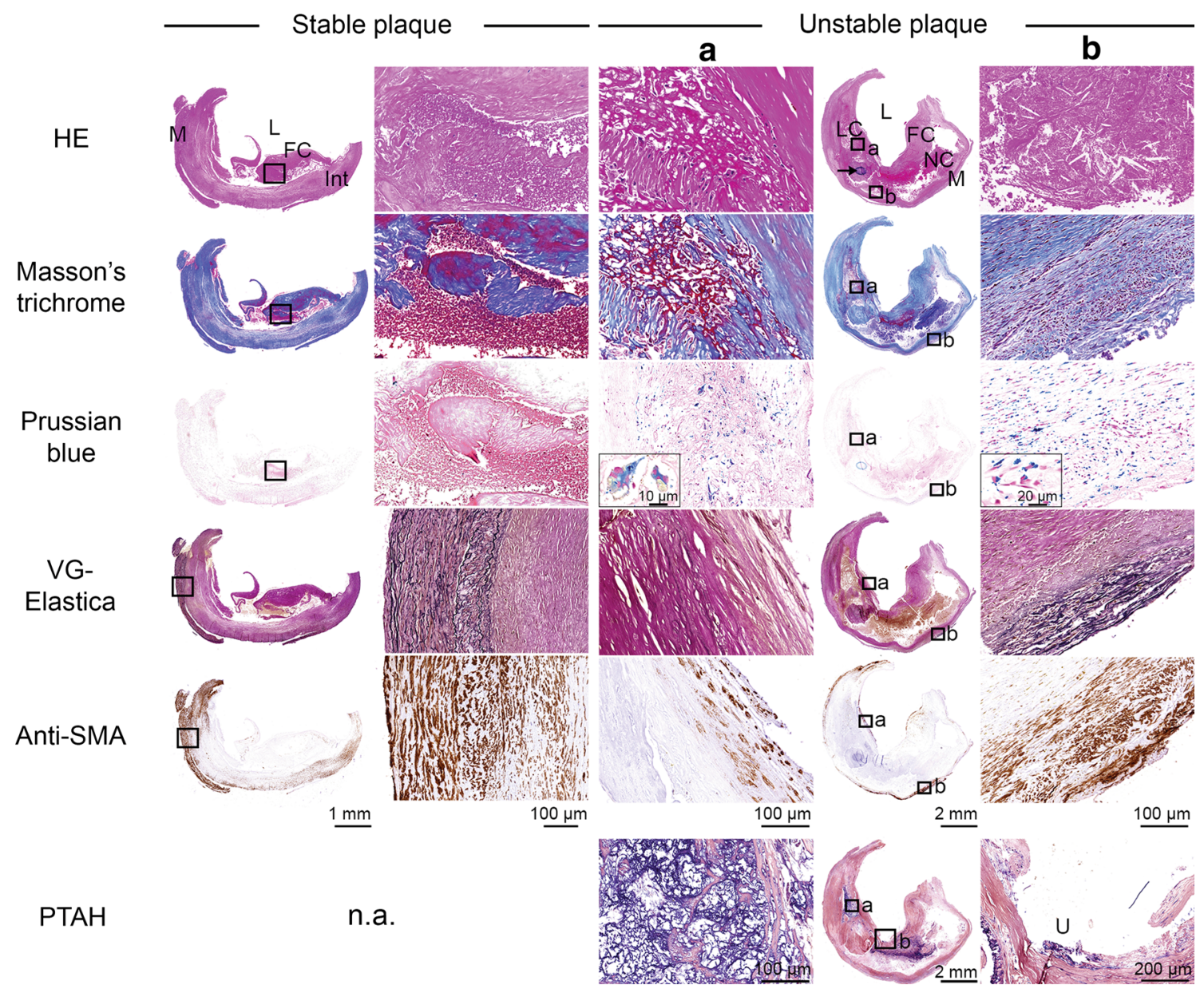

Fig. 2 Histopathological sections of the stable and unstable plaque stained with hematoxylin and eosin (HE); Masson's trichrome coloring connective tissue in blue, erythrocytes and lipid/necrotic core in intense red, smooth muscle cells in light red; Prussian blue showing iron-containing hemosiderin (blue) indicating preceding intraplaque hemorrhage; Van Gieson-Elastica (VG-Elastica) coloring lamina elastica interna and other elastic fibers in black; anti-smooth muscle cell actin (anti-SMA) antibody labeling smooth muscle cells in media and fibrous cap. Phosphotungstic acid hematoxylin staining

\section{Erythrocyte accumulation}

The gap between intima and intimal thickening/fibrous cap of the stable plaque was clearly defined in DPC and T1 contrast, but less in the other MR settings (Fig. 3a). The region was appreciated as low homogenous signal with a cloudy appearance in the zoomed DPC image (Fig. 3e). The presence of erythrocytes was only proven by histological staining.

\section{Fibrous cap}

The fibrous cap of both the stable and unstable plaque showed homogeneous and intermediate intensities in DPC images and MR images, except in MTC were it appeared hypointense (Fig. 3a, f).

(PTAH) showing fibrin in purple located in lipid and necrotic core as well as at the ulceration site. Arrow in HE-stained unstable plaque section pointing at an extensive calcification. Boxed higher magnification images of the Prussian blue stained unstable plaque show hemosiderin-loaded macrophages present in the lipid core (a) and hemosiderin-loaded smooth muscle cells present in the media (b). $L$ lumen of the blood vessel, $M$ media, Int intima, $F C$ fibrous cap, $L C$ lipid core, $N C$ necrotic core, $U$ ulceration site, n.a. not applicable

\section{Lipid core}

A lipid core was only visible in the unstable plaque composed of hemosiderin-containing macrophages and erythrocytes as well as fibrin. The lipid core appeared hypointense in T1-, T2-, DW-, and PD-weighted images, and hyperintense in MTC (Fig. 3a). The lipid core could be delineated from intima and fibrous cap in all setups. The lipid core had an inhomogeneous texture from isodense to hypodense in DPC (Fig. 3g).

\section{Necrotic core}

The necrotic core was clearly visible in all MR images with varying signal intensities, however, with more homogenous appearance than in DPC (Fig. 3a). In hypodense areas of 
A


Fig. 3 a Representative set of matched images of hematoxylin and eosin staining (HE), differential phase contrast (DPC) tomography and multicontrast MR [T1-, T2-, and proton density (PD)-weighted images, magnetization transfer contrast (MTC), diffusion-weighted (DW) images] of the stable and unstable plaque. For orientation, lumen of the blood vessel (L), media (M), intima (Int), intimal thickening (IT), fibrous cap (FC), lipid core (LC), necrotic core (NC), ulceration site $(\mathrm{U})$ are labelled. Asterisk in stable plaque indicates erythrocyte accumulation; arrowhead in unstable plaque indicates low-dense calcifications; arrow indicates high-dense calcification. b Overview section of the stable (top) and unstable (bottom) plaque with boxed regions of interest for high-power magnification images in c-k. c-k Histopathology (HE-stained) and corresponding DPC tomograms of different plaque components of the stable $(\mathbf{c}-\mathbf{e})$ and unstable (f-k) plaque the DPC images we identified cholesterol crystals or dissolved crystals and in hyperdense regions cell debris were identified (Fig. 3h, i, respectively).

\section{Calcifications}

The highest and distinct signal intensity of all plaque components was found in regions of calcification in the unstable plaque (Fig. 3a, j). High-dense calcification (high amount of calcium, arrow) appeared hyperdense/intense in DPC and MTC. In T1-, T2-, DW- and PDweighted images it appeared hypointense. This calcification was sharply delineated from the surrounding soft tissue. In contrast, low-dense calcification (arrowhead) was distinguished only on T1-weighted images as hyperintense.

\section{Ulceration}

The focal ulceration site in the unstable plaque was fibrinpositive and appreciated as a ruptured cap in HE and DPC (Fig. 3k) and was not clearly discernable in MR images.

\section{Three-dimensional visualization of endarterectomized atherosclerotic plaques in DPC}

The three-dimensional rendering of DPC images of the carotid plaque specimens are shown in Fig. 4. The needleshaped crystal in the unstable plaque appeared, in the given field of view, with an approximate length of $1.5 \mathrm{~mm}$ (Fig. 4e). Due to the limited field of view in DPC tomography only a part of the calcified crystal was imaged, 
Table 1 Morphologic characteristics of plaque components compared with appearance in DPC and MRI

\begin{tabular}{|c|c|c|c|c|c|c|}
\hline Components & DPC & $\mathrm{T} 1$ & $\mathrm{~T} 2$ & PD & MTC & DW \\
\hline Media (stable) & Isodense & Hyperintense & Isointense & Isointense & Isointense & Hyperintense \\
\hline $\begin{array}{l}\text { Media with hemosiderin } \\
\quad \text { (unstable) }^{\mathrm{a}}\end{array}$ & Isodense & Hypointense & Hypointense & Hypointense & Hyperintense & Hypointense \\
\hline Intima & Isodense & $\begin{array}{l}\text { Hyper- to } \\
\text { isointense }\end{array}$ & Isointense & Isointense & Hypointense & Hyperintense \\
\hline Intimal thickening & Hyperdense & Isointense & Isointense & Isointense & Hypointense & Isointense \\
\hline Fibrous cap & Isodense & Isointense & Isointense & Isointense & Hypointense & Isointense \\
\hline Lipid core & $\begin{array}{l}\text { Iso- to } \\
\text { hypodense }\end{array}$ & $\begin{array}{l}\text { Iso- to } \\
\text { hypointense }\end{array}$ & Hypointense & $\begin{array}{l}\text { Iso- to } \\
\text { hypointense }\end{array}$ & Hyperintense & Hypointense \\
\hline Necrotic core & $\begin{array}{l}\text { Hyper- to } \\
\text { hypodense }\end{array}$ & Isointense & $\begin{array}{l}\text { Hyper- to } \\
\text { hypointense }\end{array}$ & $\begin{array}{l}\text { Iso- to } \\
\text { hypointense }\end{array}$ & Isointense & $\begin{array}{l}\text { Hyper- to } \\
\text { hypointense }\end{array}$ \\
\hline \multicolumn{7}{|l|}{ Calcifications } \\
\hline Low-dense & Isodense & Hyperintense & Isointense & Isointense & Isointense & Isointense \\
\hline High-dense & Hyperdense & Hypointense & Hypointense & Hypointense & Hyperintense & Hypointense \\
\hline
\end{tabular}

${ }^{a}$ Media of the unstable plaque contained iron-containing hemosiderin engulfed by SMCs attributable for intense signals in MRI

however, allowing estimating that a relatively large calcification was present in this plaque specimen.

\section{Discussion}

In this ex vivo feasibility study we were able to demonstrate that detailed information on morphological characteristics and composition of a human stable and unstable endarterectomized plaque can be obtained with DPC tomography and multicontrast MRI. Our observations are in good correlation with histopathology.

Assessment of anatomy and composition of atherosclerotic plaques by MRI have been extensively studied ex vivo [8, 18-21] and in vivo [7, 22-24] using animal models of disease and in patients. However, a comprehensive evaluation of different MRI sequences has not been determined. With our combination of high-resolution MRI sequences, we were able to accurately identify fibrocellular tissue, lipid-rich and calcified regions as well as iron-containing deposits in the atherosclerotic plaques. Our intension was to apply the findings of MR images and histopathology to understand appearances of plaque characteristics in DPC images. In general, the DPC images presented a lower contrast than MR images, however, homo- and heterogeneous textures within the plaques are more pronounced in DPC images. All fibrocelluar tissue, e.g. media, fibrous cap and intimal thickening, was recognized as a homogeneous pattern and delimited to the plaque. Regions of active remodeling within the unstable plaque (lipid/necrotic core) were clearly recognized as areas with heterogeneous appearance and different signal intensities. High-dense, but not low-dense, calcification gave a distinct hyperdense signal that clearly allowed for differentiation from background structures. Contrary to MRI, hemosiderin deposits presented no significant signal in DPC. There are a few studies [25-29] reporting on the application of phase contrast imaging to atherosclerotic vessels and findings are in good agreement with our results.

With our study we provided a foundation for directing and validating the interpretation of MR and DPC images and attempted to develop and optimize imaging parameters. The relatively new technique, DPC tomography was conducted at a synchrotron radiation facility, which, so far, cannot be used in clinical practice and limits it to a benchmarking feasibility work. Recording one tomogram with the experimental parameters used in this study would result in a deposition of approximately 30,000 Gy [4]. For humans, a whole-body irradiation of up to $1 \mathrm{~Gy}$ is unlikely to cause long range symptoms, whereas a dose of $>30 \mathrm{~Gy}$ is always fatal [30]. MRI has distinct advantages over DPC including no ionizing radiation and it can be applied in vivo [31] and hence to e.g. monitor plaque progression in longitudinal studies.

There are a number of limitations to this proof-of-concept study. First, the low sample number of only one stable and one unstable carotid plaque prevent us to raise quantitative data about the sensitivity, specificity and accuracy of DPC tomography and MRI. In this present study we primarily focused on finding optimal imaging parameters receiving best image quality and contrast. With this we gained information on how to understand and interpret the signals and contrasts of the different plaque components in DPC and multicontrast MRI. Second, assessment of sensitivities for different lesion components in DPC and MRI was done retrospectively. For further studies, the 


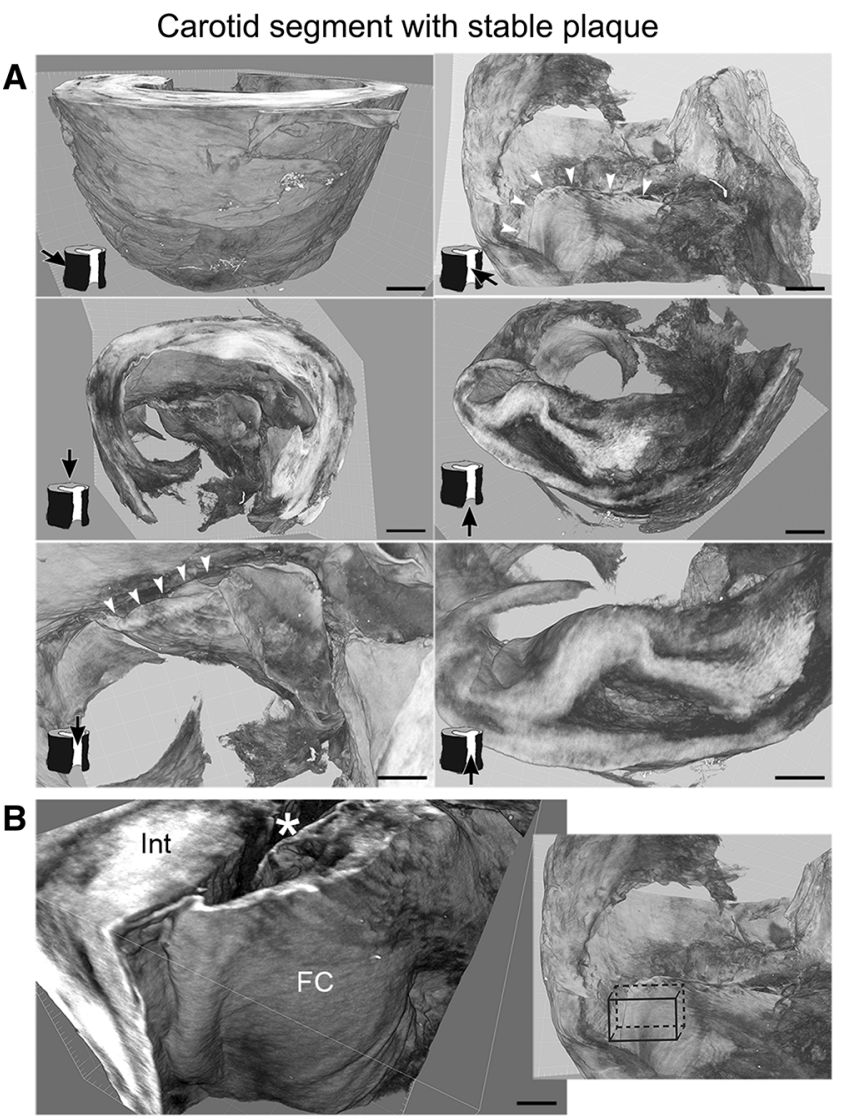

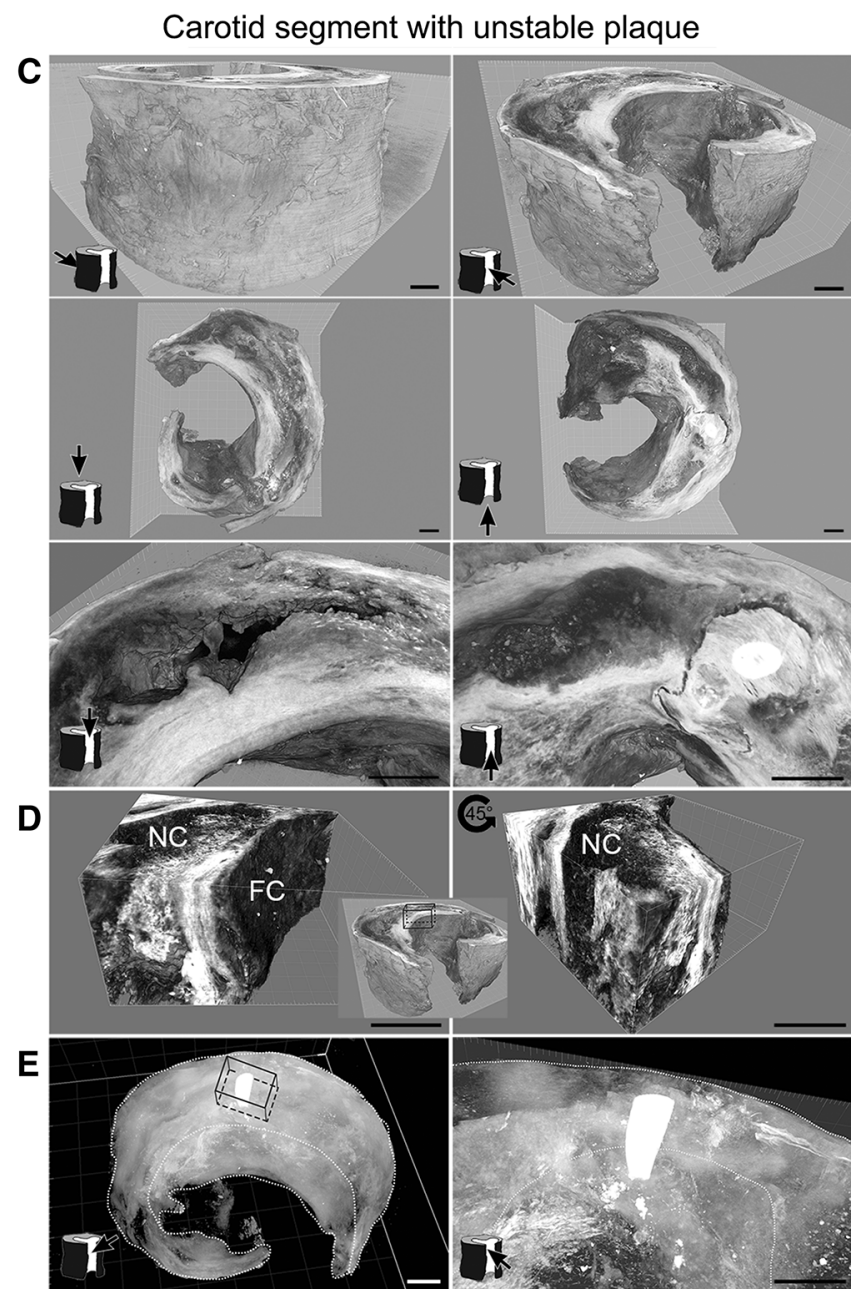

with the unstable plaque at different orientations; scale bars $1 \mathrm{~mm}$. d Cropped region of the unstable plaque showing thin fibrous cap (FC) covering the necrotic core (NC); scale bars $0.5 \mathrm{~mm}$. e Left image showing the whole unstable plaque in a maximal intensity projection. Right image showing cropped region with calcified crystal; scale bars $1 \mathrm{~mm}$

images and can discriminate between relevant components of the atherosclerotic vessel wall. Once adequately validated and optimized, DPC tomography might potentially help to define high-risk atherosclerotic plaques. Finally, we want to emphasize the importance of multicontrast imaging since only one contrast cannot differentiate all components. However, using multicontrast MRI will also not allow discriminating each single component, e.g. calcification and iron deposits displayed the same MR signal. More sophisticated diagnostic tools (e.g. new MR protocols) and validation studies will help understanding different contrasts. In this present feasibility study DPC and MRI imaging protocols for human carotid plaques were optimized and validated with a small sample size. This work will help to design and conduct further studies with higher 
numbers of plaques to obtain quantitative information on plaque morphology at statistical significance.

Acknowledgments The authors are grateful to Dr. Bernd R. Pinzer and Sabina Wunderlin for technical support. The Scientific Center for Optical and Electron Microscopy (ScopeM) of the ETH Zurich is acknowledged for support. We thank the surgeon Zoran Rancic (Z.R.) from the Clinic for Cardiovascular Surgery, University Hospital Zurich, for the initial macroscopic classification of the plaques. The team of Prof. Philipp A. Kaufmann from the Department of Nuclear Medicine, Cardiac Imaging, University Hospital Zurich, is acknowledged for coordinating the plaque collection.

\section{Compliance with Ethical Standards}

\section{Conflict of interest None.}

Funding This work was financially supported by the Clinical Research Priority Program (CRPP) of the University of Zurich on Molecular Imaging (MINZ) and the Swiss National Science Foundation (Grant PZ00P3_136822 to J.K.).

\section{References}

1. World Health Organization, Mackay J, Mensah GA, Mendis S, Greenlund K (2004) The atlas of heart disease and stroke. World Health Organization, Geneva

2. Go AS, Mozaffarian D, Roger VL, Benjamin EJ, Berry JD, Blaha MJ, Dai S, Ford ES, Fox CS, Franco S, Fullerton HJ, Gillespie C, Hailpern SM, Heit JA, Howard VJ, Huffman MD, Judd SE, Kissela BM, Kittner SJ, Lackland DT, Lichtman JH, Lisabeth LD, Mackey RH, Magid DJ, Marcus GM, Marelli A, Matchar DB, McGuire DK, Mohler ER, III, Moy CS, Mussolino ME, Neumar RW, Nichol G, Pandey DK, Paynter NP, Reeves MJ, Sorlie PD, Stein J, Towfighi A, Turan TN, Virani SS, Wong ND, Woo D, Turner MB, American Heart Association Statistics C, Stroke Statistics S (2014) Heart disease and stroke statistics-2014 update: a report from the American Heart Association. Circulation 129:e28-e292. doi:10.1161/01.cir.0000441139.02102.80

3. Finn AV, Nakano M, Narula J, Kolodgie FD, Virmani R (2010) Concept of vulnerable/unstable plaque. Arterioscler Thromb Vasc Biol 30:1282-1292. doi:10.1161/ATVBAHA.108.179739

4. Pinzer BR, Cacquevel M, Modregger P, McDonald SA, Bensadoun JC, Thuering T, Aebischer P, Stampanoni M (2012) Imaging brain amyloid deposition using grating-based differential phase contrast tomography. Neuroimage 61:1336-1346. doi:10. 1016/j.neuroimage.2012.03.029

5. Stampanoni M, Groso A, Isenegger A, Mikuljan G, Chen Q, Bertrand A, Henein S, Betemps R, Frommherz U, Bohler P, Meister D, Lange M, Abela R (2006) Trends in synchrotronbased tomographic imaging: the SLS experience. Dev X-Ray Tomogr 5:6318. doi:10.1117/12.679497

6. Weitkamp T, Diaz A, David C, Pfeiffer F, Stampanoni M, Cloetens P, Ziegler E (2005) X-ray phase imaging with a grating interferometer. Opt Express 13:6296-6304

7. Toussaint JF, LaMuraglia GM, Southern JF, Fuster V, Kantor HL (1996) Magnetic resonance images lipid, fibrous, calcified, hemorrhagic, and thrombotic components of human atherosclerosis in vivo. Circulation 94:932-938

8. Shinnar M, Fallon JT, Wehrli S, Levin M, Dalmacy D, Fayad ZA, Badimon JJ, Harrington M, Harrington E, Fuster V (1999) The diagnostic accuracy of ex vivo MRI for human atherosclerotic plaque characterization. Arterioscler Thromb Vasc Biol 19:27562761

9. Cai JM, Hatsukami TS, Ferguson MS, Small R, Polissar NL, Yuan C (2002) Classification of human carotid atherosclerotic lesions with in vivo multicontrast magnetic resonance imaging. Circulation 106:1368-1373

10. Qiao Y, Ronen I, Viereck J, Ruberg FL, Hamilton JA (2007) Identification of atherosclerotic lipid deposits by diffusionweighted imaging. Arterioscler Thromb Vasc Biol 27:14401446. doi:10.1161/ATVBAHA.107.141028

11. Qiao Y, Hallock KJ, Hamilton JA (2011) Magnetization transfer magnetic resonance of human atherosclerotic plaques ex vivo detects areas of high protein density. J Cardiovasc Magn Reson 13:73. doi:10.1186/1532-429X-13-73

12. Müller A, Mu L, Meletta R, Beck K, Rancic Z, Drandarov K, Kaufmann PA, Ametamey SM, Schibli R, Borel N, Krämer SD (2014) Towards non-invasive imaging of vulnerable atherosclerotic plaques by targeting co-stimulatory molecules. Int J Cardiol 174:503-515. doi:10.1016/j.ijcard.2014.04.071

13. Stary HC, Chandler AB, Dinsmore RE, Fuster V, Glagov S, Insull W Jr, Rosenfeld ME, Schwartz CJ, Wagner WD, Wissler RW (1995) A definition of advanced types of atherosclerotic lesions and a histological classification of atherosclerosis. A report from the Committee on Vascular Lesions of the Council on Arteriosclerosis, American Heart Association. Arterioscler Thromb Vasc Biol 15:1512-1531

14. Saam T, Ferguson MS, Yarnykh VL, Takaya N, Xu D, Polissar NL, Hatsukami TS, Yuan C (2005) Quantitative evaluation of carotid plaque composition by in vivo MRI. Arterioscler Thromb Vasc Biol 25:234-239. doi:10.1161/01.ATV.0000149867.61851.31

15. Lacolley P, Regnault V, Nicoletti A, Li Z, Michel JB (2012) The vascular smooth muscle cell in arterial pathology: a cell that can take on multiple roles. Cardiovasc Res 95:194-204. doi:10.1093/ $\mathrm{cvr} / \mathrm{cvs} 135$

16. Riviere C, Boudghene FP, Gazeau F, Roger J, Pons JN, Laissy JP, Allaire E, Michel JB, Letourneur D, Deux JF (2005) Iron oxide nanoparticle-labeled rat smooth muscle cells: cardiac MR imaging for cell graft monitoring and quantitation. Radiology 235:959-967. doi:10.1148/radiol.2353032057

17. Tavora F, Cresswell N, Li L, Ripple M, Burke A (2010) Immunolocalisation of fibrin in coronary atherosclerosis: implications for necrotic core development. Pathology 42:15-22. doi:10.3109/00313020903434348

18. Coombs BD, Rapp JH, Ursell PC, Reilly LM, Saloner D (2001) Structure of plaque at carotid bifurcation: high-resolution MRI with histological correlation. Stroke 32:2516-2521

19. Li T, Li X, Zhao X, Zhou W, Cai Z, Yang L, Guo A, Zhao S (2012) Classification of human coronary atherosclerotic plaques using ex vivo high-resolution multicontrast-weighted MRI compared with histopathology. AJR Am J Roentgenol 198:10691075. doi:10.2214/AJR.11.6496

20. Nikolaou K, Becker CR, Muders M, Babaryka G, Scheidler J, Flohr T, Loehrs U, Reiser MF, Fayad ZA (2004) Multidetectorrow computed tomography and magnetic resonance imaging of atherosclerotic lesions in human ex vivo coronary arteries. Atherosclerosis 174:243-252. doi:10.1016/j.atherosclerosis.2004. 01.041

21. Worthley SG, Helft G, Fuster V, Fayad ZA, Fallon JT, Osende JI, Roque M, Shinnar M, Zaman AG, Rodriguez OJ, Verhallen P, Badimon JJ (2000) High resolution ex vivo magnetic resonance imaging of in situ coronary and aortic atherosclerotic plaque in a porcine model. Atherosclerosis 150:321-329

22. Gury-Paquet L, Millon A, Salami F, Cernicanu A, Scoazec JY, Douek P, Boussel L (2012) Carotid plaque high-resolution MRI at $3 \mathrm{~T}$ : evaluation of a new imaging score for symptomatic plaque 
assessment. Magn Reson Imaging 30:1424-1431. doi:10.1016/j. mri.2012.04.024

23. Worthley SG, Helft G, Fuster V, Fayad ZA, Rodriguez OJ, Zaman AG, Fallon JT, Badimon JJ (2000) Noninvasive in vivo magnetic resonance imaging of experimental coronary artery lesions in a porcine model. Circulation 101:2956-2961

24. Yuan C, Kerwin WS, Ferguson MS, Polissar N, Zhang S, Cai J, Hatsukami TS (2002) Contrast-enhanced high resolution MRI for atherosclerotic carotid artery tissue characterization. J Magn Reson Imaging 15:62-67

25. Appel AA, Chou CY, Larson JC, Zhong Z, Schoen FJ, Johnston CM, Brey EM, Anastasio MA (2013) An initial evaluation of analyser-based phase-contrast X-ray imaging of carotid plaque microstructure. Br J Radiol 86:20120318. doi:10.1259/bjr. 20120318

26. Hetterich H, Fill S, Herzen J, Willner M, Zanette I, Weitkamp T, Rack A, Schuller U, Sadeghi M, Brandl R, Adam-Neumair S, Reiser M, Pfeiffer F, Bamberg F, Saam T (2013) Grating-based $\mathrm{X}$-ray phase-contrast tomography of atherosclerotic plaque at high photon energies. Z Med Phys 23:194-203. doi:10.1016/j. zemedi.2012.12.001

27. Hetterich H, Willner M, Fill S, Herzen J, Bamberg F, Hipp A, Schuller U, Adam-Neumair S, Wirth S, Reiser M, Pfeiffer F, Saam T (2014) Phase-contrast CT: qualitative and quantitative evaluation of atherosclerotic carotid artery plaque. Radiology 271:870-878. doi:10.1148/radiol.14131554
28. Saam T, Herzen J, Hetterich H, Fill S, Willner M, Stockmar M, Achterhold K, Zanette I, Weitkamp T, Schuller U, Auweter S, Adam-Neumair S, Nikolaou K, Reiser MF, Pfeiffer F, Bamberg F (2013) Translation of atherosclerotic plaque phase-contrast CT imaging from synchrotron radiation to a conventional lab-based X-ray source. PLoS One 8:e73513. doi:10.1371/journal.pone. 0073513

29. Fujimoto S, Kondo T, Kodama T, Fujisawa Y, Groarke J, Kumamaru KK, Takamura K, Matsunaga E, Miyauchi K, Daida H, Rybicki FJ (2014) A novel method for non-invasive plaque morphology analysis by coronary computed tomography angiography. Int J Cardiovasc Imaging 30:1373-1382. doi:10. 1007/s10554-014-0461-5

30. Donnelly EH, Nemhauser JB, Smith JM, Kazzi ZN, Farfan EB, Chang AS, Naeem SF (2010) Acute radiation syndrome: assessment and management. South Med J 103:541-546. doi:10.1097/ SMJ.0b013e3181ddd571

31. Corti R, Fuster V (2011) Imaging of atherosclerosis: magnetic resonance imaging. Eur Heart J 32:1709-19b. doi:10.1093/eur heartj/ehr068 